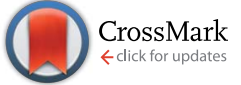

Cite this: J. Mater. Chem. A, 2016, 4 , 3278

Received 4th November 2015 Accepted 22nd January 2016

DOI: $10.1039 / \mathrm{c} 5 \mathrm{ta0} 8922 \mathrm{k}$

www.rsc.org/MaterialsA

\section{Atomically thin Pt shells on Au nanoparticle cores: facile synthesis and efficient synergetic catalysis $\uparrow$}

\begin{abstract}
C. Engelbrekt, ${ }^{a}$ N. Šešelj, ${ }^{a}$ R. Poreddy, ${ }^{b}$ A. Riisager, ${ }^{b}$ J. Ulstrup ${ }^{a}$ and J. Zhang ${ }^{\star a}$
We present a facile synthesis protocol for atomically thin platinum (Pt) shells on top of gold (Au) nanoparticles (NPs) (Au(APtNPs) in one pot under mild conditions. The Au@PtNPs exhibited remarkable stability (> 2 years) at room temperature. The synthesis, bimetallic nanostructures and catalytic properties were thoroughly characterized by ultraviolet-visible light spectrophotometry, transmission electron microscopy, nanoparticle tracking analysis and electrochemistry. The $8 \pm 2 \mathrm{~nm}$ Au@PtNPs contained $24 \pm 1 \mathrm{~mol} \% \mathrm{Pt}$ and $76 \pm 1 \mathrm{~mol} \%$ Au corresponding to an atomically thin Pt shell. Electrochemical data clearly show that the active surface is dominated by Pt with a specific surface area above $45 \mathrm{~m}^{2}$ per gram of Pt. Interactions with the Au core increase the activity of the Pt shell by up to $55 \%$ and improve catalytic selectivity compared to pure Pt. The Au@Pt NPs show exciting catalytic activity in electrooxidation of sustainable fuels (i.e. formic acid, methanol and ethanol), and selective hydrogenation of benzene derivatives. Especially high activity was achieved for formic acid oxidation, $549 \mathrm{~mA}\left(\mathrm{mg}_{\mathrm{Pt}}\right)^{-1}$ (at $0.6 \mathrm{~V}$ vs. SCE), which is 3.5 fold higher than a commercial $<5 \mathrm{~nm}$ PtNP catalyst. Excellent activity for the direct production of $\gamma$-valerolactone, an alternative biofuel/fuel additive, from levulinic acid and methyl levulinate was finally demonstrated.
\end{abstract}

\section{Introduction}

Platinum (Pt) catalysts are crucial for a range of chemical reactions in automotive, petroleum and energy conversion industries. ${ }^{1}$ Pt has excellent catalytic properties but low availability and high cost have shifted focus from pure Pt to advanced bimetallic Pt-based nanostructures.

Nanomaterial properties depend critically on chemical composition, size, shape, crystallinity, and surface structure. Bimetallic nanostructures provide a strategy for design of advanced functional nanomaterials. A second element can, for example, reduce the concentration of surface sites for catalytic poisons - the so-called third-body effect. ${ }^{2}$ Metal monolayers also have dramatically altered properties by electronic interaction with the underlying core. ${ }^{2}$ Facile synthesis of thin metal shells on top of a metal core offers attractive approaches to tuning the catalytic activity as well as efficiently utilizing Pt. $^{3}$ Gold (Au) nanostructures have been among the most studied systems due to unique optical and electronic properties, and application as catalysts for $\mathrm{CO}$ oxidation. ${ }^{4}$

Core-shell Au-Pt NPs show improved electrocatalysis, benefitting from electrochemical stability of Au, high catalytic

${ }^{a}$ NanoChemistry, Department of Chemistry, Technical University of Denmark, Kemitorvet 207, 2800, Kgs. Lyngby, Denmark. E-mail: jz@kemi.dtu.dk

${ }^{b}$ Centre for Catalysis and Sustainable Chemistry, Department of Chemistry, Technical University of Denmark, Kemitorvet 207, 2800, Kgs. Lyngby, Denmark

$\dagger$ Electronic supplementary information (ESI) available. See DOI: $10.1039 / \mathrm{c} 5 \mathrm{ta} 08922 \mathrm{k}$ activity of Pt, and a performance boost from core-shell synergy. ${ }^{5}$ $\mathrm{Au}-\mathrm{Pt}$ core-shell NPs can be prepared by precursor co-reduction followed by thermal treatment, ${ }^{6}$ by underpotential deposition (UPD) of copper $(\mathrm{Cu})$ on $\mathrm{Au}$ followed by galvanic displacement by $\mathrm{Pt}$ precursors, ${ }^{7}$ or reduction via seed-mediated growth. ${ }^{8}$ Thermally driven methods require high temperature, strong reducing agents, controlled atmosphere, or non-aqueous solvents. ${ }^{6}$ UPD is limited by the need for immobilization on conducting supports. UPD methods are superior for atomically thin Pt shells, while wet syntheses often provide thicknesses $>1$ $n{ }^{8}{ }^{8}$ Seed-based wet chemical syntheses offer potential for large-scale one-pot, green preparation, but the complexity of the processes gives poor yield and control of product morphology. Shell precursor reduction, diffusion through seed coatings, and deposition on seed surfaces all have to be controlled.9

Non-toxic procedures and sustainability have emerged as criteria in nanomaterial synthesis and applications. ${ }^{10}$ In our previous work, a starch and glucose based recipe, the saccharide-based approach to metal nanostructure synthesis (SAMENS), for the synthesis of gold nanoparticles (AuNPs) ${ }^{11}$ and other metal nanostructures was developed. A dynamics study showed that glucose acts as reducing agent and starch as capping agent in phosphate buffer. The Good's buffer 2-( $N$ morpholino)ethanesulfonic acid (MES) participates in the reduction of $\mathrm{HAuCl}_{4}$ and adsorbs on the AuNP surface as well as controlling $\mathrm{pH} .{ }^{12}$ Very small $(1.6-1.8 \mathrm{~nm})$ catalytically active starch coated platinum nanoparticles (PtNPs) have also been produced by the SAMENS recipe. ${ }^{13}$ In the present study we have 
explored SAMENS for the synthesis of stable and uniform Au-Pt bimetallic nanostructures with high catalytic activity. Uniform AuNPs serve as seeds for Au-Pt core-shell (Au@Pt) NPs with atomically thin Pt shells. Ultraviolet-visible light spectroscopy (UV-vis), NP tracking analysis (NTA), transmission electron microscopy (TEM) and electrochemical methods were employed to characterize the synthesis, structure, and properties. A catalyst of well dispersed Au@PtNPs with 12 wt\% metal (3 wt\% Pt, $9 \mathrm{wt} \% \mathrm{Au}$ ) on graphitized carbon black (G-CB) was prepared and comprehensively characterized. Starch capping was successfully removed thermally and the catalytic activity investigated by electrocatalytic oxidation of small organic fuel molecules, and by selective aromatic ring hydrogenation (ARH) processes and hydrocyclization of levulinic acid (LA) and methyl levulinate (MLA).

Small organic molecules such as formic acid (FA), methanol $(\mathrm{MeOH})$, and ethanol (EtOH), have been widely used for fuel cell (FC) systems as these fuels can be produced from renewable sources without harmful waste. FA is attractive since it is nontoxic and can be used at high concentrations due to low rates of crossing commonly used membranes. This leads to good FC performance despite the low energy density compared to $\mathrm{MeOH}$ and $\mathrm{EtOH}$. FA oxidation entails no $\mathrm{C}-\mathrm{C}$ bond breaking which is a serious obstacle for EtOH oxidation. $\mathrm{MeOH}$ has received much attention as a $\mathrm{C}_{1}$ fuel with high energy density but faces challenges of toxicity and membrane crossing. EtOH exhibits low crossover and as a potential 12-electron oxidation process has a very high energy density. ${ }^{14}$ The Au@PtNP catalysts exhibit high specific electrochemical Pt surface area (ECSA) and excellent catalytic activity for all the FA (FAOR), $\mathrm{MeOH}$ (MOR) and EtOH (EOR) oxidation reactions. Currents reach 549, 219 and $229 \mathrm{~mA}\left(\mathrm{mg}_{\mathrm{Pt}}\right)^{-1}$ for $\mathrm{FA}, \mathrm{MeOH}$ and EtOH, respectively, in $0.1 \mathrm{M}$ fuel (scan rate $50 \mathrm{mV} \mathrm{s}^{-1}$ ), or 3.5, 2.0 and 3.1 times that of a $<$ $5 \mathrm{~nm}$ commercial PtNP catalyst (Sigma). Apart from improved Pt utilization in the atomically thin shell, synergy between the Pt shell and the Au core improves the Pt surface activity by as much as $55 \%$ relative to pure $\mathrm{Pt}$.

ARH to cyclohexane derivatives is important in petrochemical and pharmaceutical industry. ${ }^{15,16}$ Chemoselective ARH of benzoic acid (BA) to cyclohexane carboxylic acid (CCA) is crucial in the synthesis of $\varepsilon$-caprolactam, ${ }^{17}$ but $\mathrm{ARH}$ requires harsh conditions with a suitable catalyst to selectively hydrogenate the ring. Noble metal catalysts ( $\mathrm{Ru}-\mathrm{Pt},{ }^{18} \mathrm{Rh} / \mathrm{C},{ }^{19} \mathrm{Pd} / \mathrm{C},{ }^{17,20} \mathrm{Pd}-\mathrm{Ru}^{21}$ ) have been extensively studied. $\mathrm{Pd} / \mathrm{C}$ is traditionally used though there are a number of limitations for ARH of BA to CCA, i.e. fast deactivation, and need for high temperature $(423 \mathrm{~K})$ and high $\mathrm{H}_{2}$ pressure (15 MPa). ${ }^{22-24}$ Recently, Pt was reported as an active ARH catalyst with activity/selectivity depending strongly on NP shape and size. ${ }^{25}$

Catalytic conversion of cellulosic biomass to various platform molecules, e.g. $\gamma$-valerolactone (GVL), is a key step in methyltetrahydrofuran production. GVL can be obtained by hydrocyclization of LA, an inexpensive starting material obtainable from biomass. Multiple catalytic strategies to produce LA via biomass transformation are available, ${ }^{26}$ but efficient conversion of LA to GVL using green technology is warranted. To date, numerous supported metal catalysts have been employed to hydrogenate LA to GVL with Ru-based catalysts being superior. ${ }^{27,28} \mathrm{Pt}$ is active for LA hydrogenation, but activity comparable to that of Ru using green technologies is yet to be obtained.

The Au@Pt catalyst introduced in this work efficiently and selectively catalyzes the ARH of BA and benzamide (BM), with high activity (TOF $=62 \mathrm{~h}^{-1}$ ) for GVL synthesis from both LA and MLA. To the best of our knowledge, ARH of BA and its derivatives, and catalytic production of GVL from LA/MLA over Au-Pt catalysts has not been reported before.

\section{Experimental section}

\section{Au-Pt core-shell nanoparticles (Au@Pt NPs) with atomically thin Pt shells}

5-200 mL AuNP seed solution was prepared by pre-heating a MES buffered mixture of glucose and starch for 5-10 min in a $95{ }^{\circ} \mathrm{C}$ water or oil bath, adding gold precursor, and allowing 30 min for AuNP formation. The AuNP seed solution contained 2 $\mathrm{mM} \mathrm{HAuCl}_{4}, 10 \mathrm{mM}$ glucose, $0.6 \mathrm{wt} \%$ starch and $10 \mathrm{mM}$ MES ( $\mathrm{pH}$ 7). Following AuNP formation, the seed solution was diluted 4 times with water and $20 \mathrm{mM} \mathrm{H}_{2} \mathrm{PtCl}_{6}$ was added to obtain a $\mathrm{Au}$ : Pt mol ratio of $5: 2$ while heating was maintained for another 1-2 $\mathrm{h}$. Complete reduction of the Pt precursor was confirmed with UV-vis. The original seed solution had a deep red colour while the final core-shell nanoparticle solution was dark brown.

\section{Electrocatalytic oxidation}

Details about electrochemical measurements are given in the supplementary information. After potential stabilization and $\mathrm{CV}$ in $0.1 \mathrm{M} \mathrm{H}_{2} \mathrm{SO}_{4}$, formic acid (FA), methanol (MeOH) or ethanol (EtOH) were admitted into the cell to obtain a concentration of $0.1 \mathrm{M}$. All the experiments were performed at room temperature (r.t.).

\section{Catalytic hydrogenation}

A detailed description of hydrogenation experiments is given in the supplementary information. In brief, chemoselective aromatic hydrogenation of BA and BM, and cyclohydrogenation of LA and MLA was conducted in a $100 \mathrm{~mL}$ stainless steel autoclave reactor (Fig. $\mathrm{S} 1 \dagger$ ). Substrate, solvent, and catalyst were mixed in the autoclave, the reactor evacuated and pressurized with dihydrogen, heated to the target temperature, and maintained for 0.5 to $24 \mathrm{~h}$. After cooling to r.t., aliquots of the reaction mixture were submitted to gas chromatography (GC), and the structure of the products identified using GC coupled with mass spectrometry (GC-MS).

\section{Results and discussion}

Controlling the size of the Au core allows tuning of the core-shell nanostructure properties. AuNPs in the range of 8-80 nm were successfully prepared using a seeded growth approach inspired by Bastus et al. ${ }^{29}$ The synthesis procedure and AuNP characterization are provided in the supplementary information, 

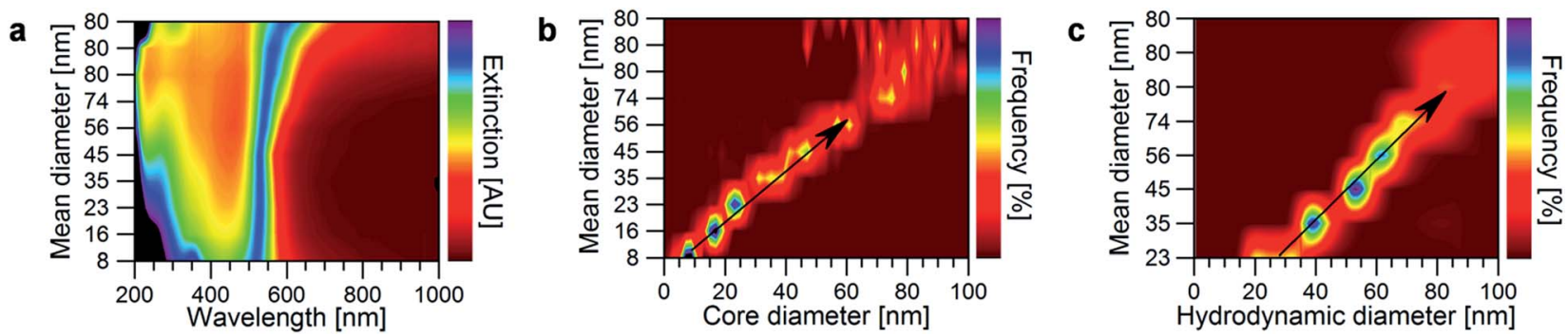

Fig. 1 Size control of AuNP cores. 3D plots of (a) extinction, (b) core diameter and (c) hydrodynamic diameter of the 10 partitions. Plots for individual partition are stacked along the $y$-axis with extinction (in a) and frequency (in b and c) represented with the colour scheme from 0 (black) to max (purple). The UV-vis spectra were normalized at the LSPR max. The $y$-axis labels represent the size of the AuNPs derived from TEM images. UV-vis spectra and size distributions for the individual fractions are provided in Fig. S2 and S3.广

Fig. S2-S6 and Tables S1-S2.† Fractions were obtained at various phases of the synthesis and characterized by UV-vis, TEM and NTA (Fig. 1a-c, respectively). A systematic increase in size was confirmed with stable NP dispersions in the range of 8-80 $\mathrm{nm}$. With increasing particle size, the localized surface plasmon resonance (LSPR) band initially narrowed followed by red-shifting and broadening. Above $80 \mathrm{~nm}$ the dispersions became unstable leading to aggregation due to a thinning of the starch capping with increasing size as seen by comparing TEM and NTA data.

\section{Synthesis of Au@Pt core-shell NPs}

The two-step, one-pot synthesis strategy for the preparation of $\mathrm{Au} @ P t N P$ f from the SAMENS method is illustrated in Fig. 2a. The AuNP cores were first prepared by chemical reduction of $\mathrm{HAuCl}_{4}$ in buffered glucose-starch solution. ${ }^{12}$ Secondly, an atomically thin Pt shell was formed by reduction of $\mathrm{H}_{2} \mathrm{PtCl}_{6}$ to provide Au@Pt core-shell NPs. The precursor was reduced directly after the formation of AuNPs with the temperature maintained at $95{ }^{\circ} \mathrm{C}$. The structures of the synthesis components are given in Scheme S1. $\dagger$ Formation of $\mathrm{Au}$ and Pt bimetallic NPs requires control of precursor reduction. Too fast reduction of the $\mathrm{Pt}$ precursor leads to formation of separate PtNPs. Characteristic UV-vis spectra of the SAMENS prepared Au@PtNPs, PtNPs and AuNPs are shown in Fig. 2b. The extinction from the Au@PtNPs (a continuum with a weak shoulder around $500 \mathrm{~nm}$ ) resembles that of PtNPs with an indication of spectral features from AuNPs, indicating a drastic change in the AuNP surface structure. No unreacted metal precursors could be detected.

More details about the change in optical properties of the $\mathrm{Au} @ P t N P s$ were obtained by varying the concentration of the Pt precursor. 0.2, 0.5 and $1.0 \mathrm{mM}$ Pt precursor (AuPt-0.2, - 0.5 and -1.0 , respectively) was employed while keeping the concentration of Au fixed at $0.5 \mathrm{mM}$. Fig. S7† shows UV-vis spectra of the three samples after terminated heating (solid lines) and 2 years of storage at r.t. (dotted lines). The lowest Pt precursor concentration led to a dramatic broadening of the LSPR peak of the AuNP core strongly indicative of formation of a Pt shell around the AuNPs. This damping by deposited Pt was previously reported by Henglein when reducing Pt precursor with hydrogen gas. ${ }^{30}$ No significant LSPR damping was observed with higher Pt concentrations after terminated heating or even after complete Pt precursor reduction. Under these conditions, separate PtNPs rather than core-shell NPs were formed and the
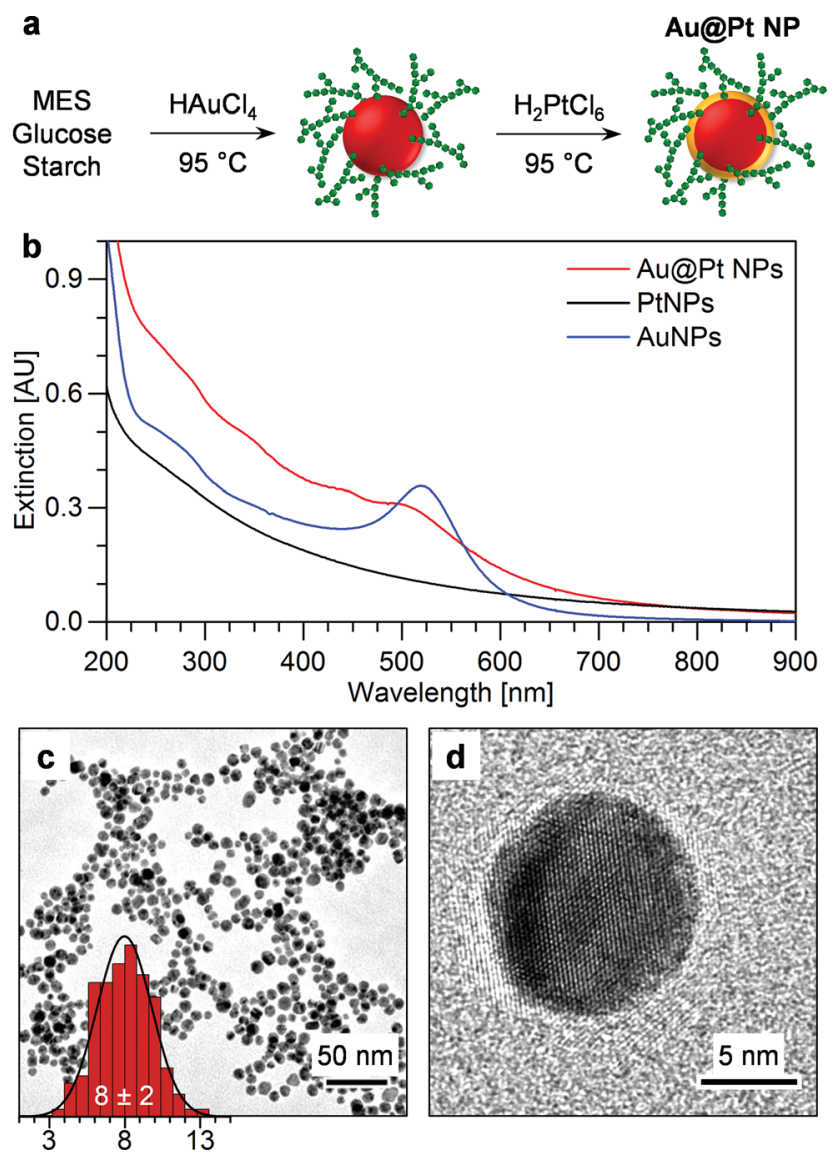

Fig. 2 (a) Principle of synthesis of Au@Pt NPs. AuNP cores (red) coated by starch (green) are synthesized and the Pt shell (orange) of the Au@Pt NPs subsequently formed in the same pot. (b) Extinction spectra of assynthesized Au(Pt NPs (red), PtNPs (black) and AuNPs (blue) from the SAMENS method showing almost complete LSPR quenching by the Pt shell. (c) TEM micrograph of as-synthesized Au@Pt NPs. No evidence of separate PtNPs was observed. Insert in (c) shows the size distribution in $\mathrm{nm}$ of the NPs measured from TEM images. (d) HR-TEM of assynthesized Au@Pt nanostructures showing no indication of a Pt shell despite consistent Pt signal in EDX measurements. 
colloids retained the characteristic red colour of the AuNPs, Fig. S8. $\dagger$ All NP dispersions remain stable after more than 2 years at r.t. owing to the efficient steric stabilization by the starch coating. The LSPR peak of the AuNP core grew slightly over time due to rearrangement of the Pt shell, Fig. S9. $\dagger$

TEM was used to evaluate the size and elemental composition of the Au@PtNPs, Fig. 2c and d. The size of the AuNP seeds was $8 \pm 2 \mathrm{~nm}$, which was also found for Au@PtNPs. The size of the freshly formed AuNPs and AuNPs which had undergone cooling and reheating (to simulate the $\mathrm{Pt}$ shell formation process) was measured to $8 \pm 2$ and $7 \pm 2 \mathrm{~nm}$, respectively (Fig. S10†). In all cases, no significant change in size was identified. Energy dispersive X-ray spectroscopy (EDX) confirmed that the Au@PtNPs contained both $\mathrm{Au}$ and Pt, Fig. 3a, at $76 \pm 1$ and $24 \pm 1 \mathrm{~mol} \%$, respectively (based on 12 EDX measurements).

This ratio was also found for Au@PtNP samples in which the Pt shell was produced immediately after AuNP formation. The Pt shell thickness was estimated based on ensemble EDX measurements assuming perfectly spherical $8 \mathrm{~nm}$ particles (as measured from TEM) with ideal core-shell geometry to $3 \AA$. This was substantiated by individual NP EDX data providing a shell thickness of $2.9 \pm 0.7 \AA$, Fig. 3b, which fits with a single Pt atom $(2.77 \AA)$. In fact, scanning transmission electron microscopy with EDX (STEM-EDX) of single NPs shows evenly distributed $\mathrm{Pt}$ and that Pt signals dominate around the NP perimeters, Fig. 4.

Further evidence of a Pt shell was found in the electrochemical response of the bimetallic NPs. Cyclic voltammetry (CV) of the Au@PtNPs drop cast on glassy carbon electrodes

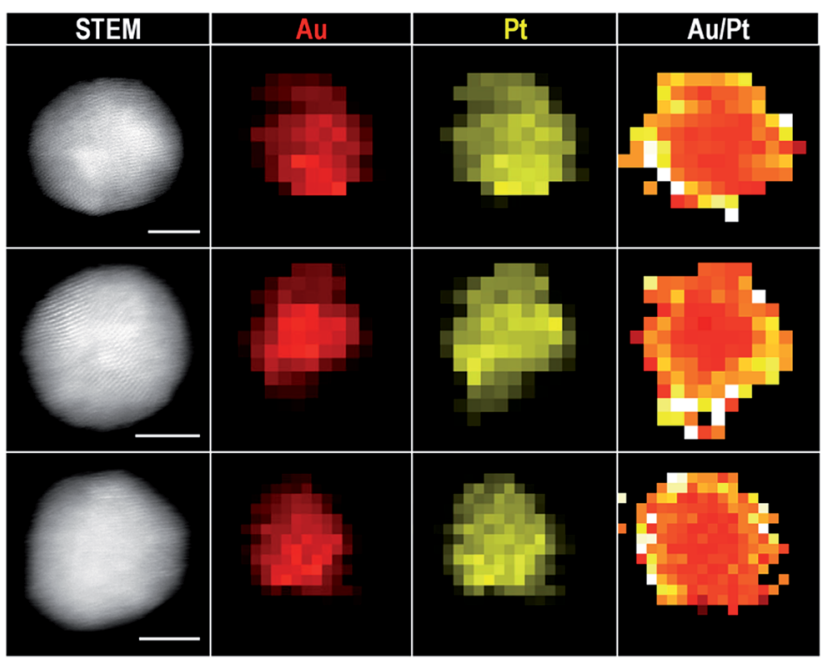

Fig. 4 Elemental mapping of individual Au@PtNPs by STEM-EDX. High-resolution STEM images are shown in the left column, elemental maps of Au and Pt in the middle columns, and a Au/Pt signal ratio map in the right column (white/yellow = high $\mathrm{Pt} / \mathrm{Au}$, red = low $\mathrm{Pt} / \mathrm{Au}$ ). Scale bars correspond to $3 \mathrm{~nm}$.

revealed broad anodic signals from 0.6 to $1.3 \mathrm{~V}$ from oxidation of the Pt shell and uncovered areas of the Au core, and clear cathodic peaks corresponding to the reduction of $\mathrm{PtO}_{x}(0.36 \mathrm{~V})$ and $\mathrm{AuO}_{x}(0.86 \mathrm{~V})$ showing that both metals are present at the surface, Fig. 3c. The oxide reductions in the bimetallic NPs accord with observations for pure SAMENS Au and Pt NPs tested under the same conditions (Fig. 3d) though the reduction
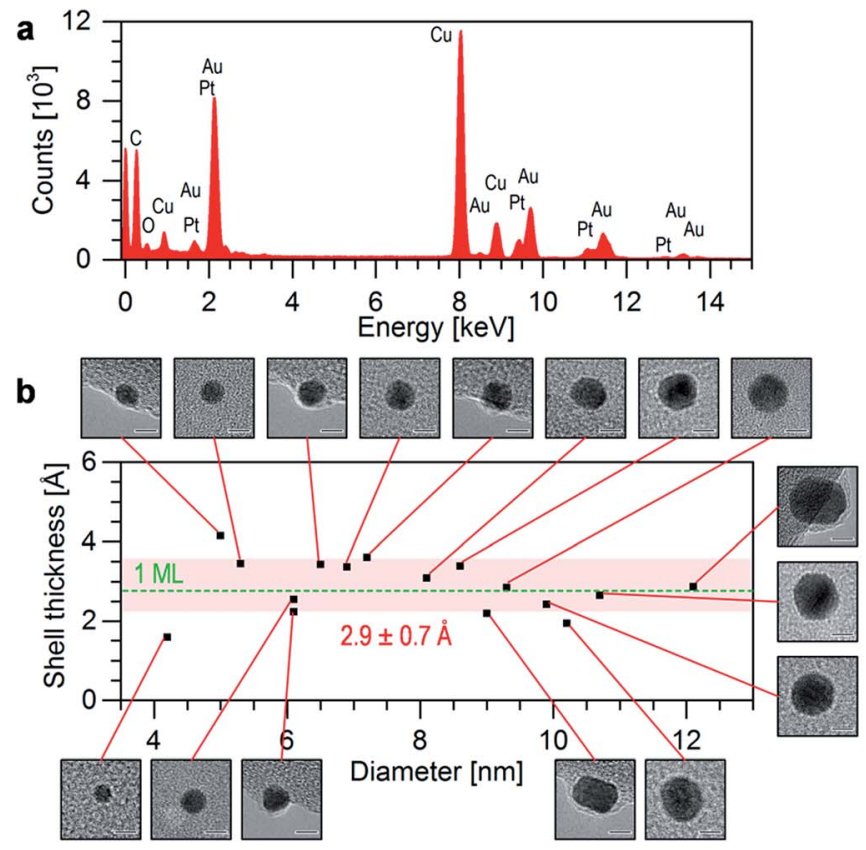

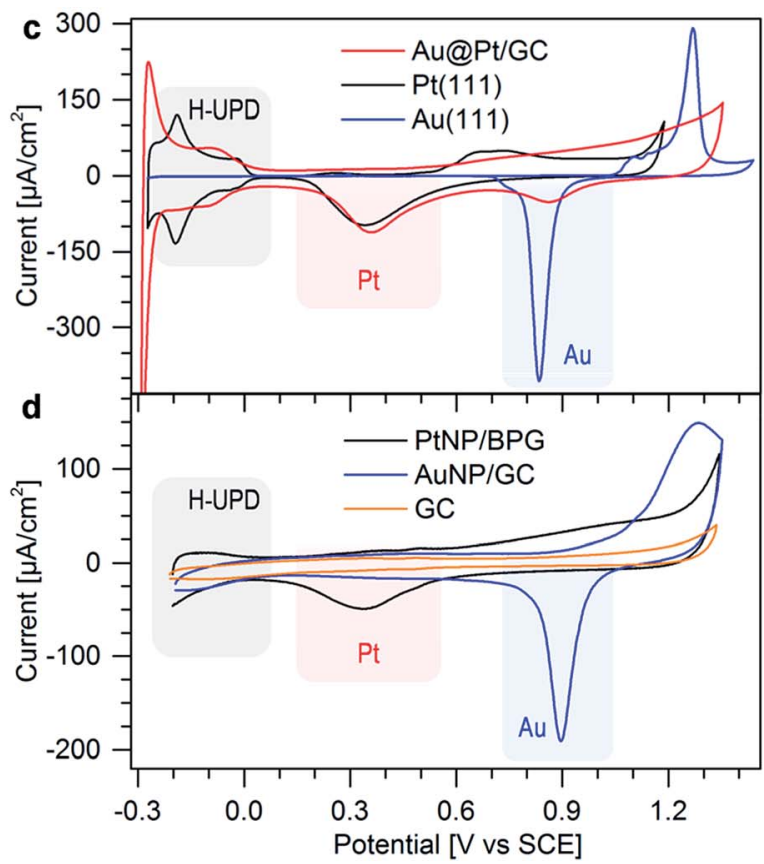

Fig. 3 Identification of the Pt shell. (a) EDX spectrum of as-synthesized Au@Pt NPs. (b) Estimated Pt shell thickness based on EDX and HR-TEM images of individual Au@Pt NPs. Red band shows mean \pm 1 standard deviation and the green dashed line the diameter of metallic Pt. CVs of (c) as-synthesized Au@Pt NPs, single-crystal Pt and $\mathrm{Au}$, (d) CVs of as-synthesized monometallic NPs and bare glassy carbon $\left(0.1 \mathrm{M} \mathrm{H}_{2} \mathrm{SO}_{4}, \mathrm{RE}=\mathrm{RHE}\right.$, $\mathrm{CE}=\mathrm{Pt}$, scan rate $=50 \mathrm{mV} \mathrm{s}^{-1}$ ). Pt and Au oxide reduction and hydrogen adsorption/desorption are highlighted. 
potentials are shifted slightly, Table S3. $\dagger$ The Au oxide reduction is shifted $40 \mathrm{mV}$ negatively suggesting that $\mathrm{Au}$ oxide is stabilized by the Pt shell whereas the Pt oxide reduction is shifted $30 \mathrm{mV}$ positively. The hydrogen adsorption/desorption region at low potentials is characteristic of $\mathrm{Pt}$ and further supports the abundance of exposed metallic Pt.

\section{Catalyst preparation and characterization}

The Au@PtNPs were immobilized on a graphitized carbon black support (G-CB), which is robust against degradation due to a high content of $\mathrm{sp}^{2}$ carbon..$^{31}$ Good wetting of the support was ensured by ultrasonication, prolonged stirring and heating in water prior to mixing with as-synthesized Au@PtNPs. The G-CB suspension was added to the Au@PtNP colloid shortly after Pt shell formation, and adsorption completed within $1 \mathrm{~h}$, Fig. S11. $\dagger$ The starch coating used in this work was not covalently bound to the NP surface and could be removed via oxidation by heating in air at $300{ }^{\circ} \mathrm{C}$ for $1 \mathrm{~h}$. Fig. S12a $\uparrow$ shows thermogravimetric analysis (TGA) confirming that starch began decomposing/oxidizing around $200{ }^{\circ} \mathrm{C}$, while the bare G-CB support was stable up to $700{ }^{\circ} \mathrm{C}$. TGA of the Au@Pt/G-CB before and after thermal treatment are identical except for the lowtemperature region corresponding to mass loss from starch and no indication of starch was found after thermal treatment. Oxidation of the carbon support was catalyzed by the $\mathrm{Au} @$ PtNPs. The onset of oxidation thus shifted from 730 to $570{ }^{\circ} \mathrm{C}$ in the presence of the bimetallic NPs. The oxidation of the starch capping was also catalyzed by the Au@PtNPs as indicated by shifts of the two exothermic peaks in the differential thermal analysis (DTA) of starch from $300 / 390{ }^{\circ} \mathrm{C}$ to 190/260 ${ }^{\circ} \mathrm{C}$, Fig. S12b. $\dagger$ The masses finally obtained were assigned to the adsorbed Au@PtNPs and corresponded to 12-13 wt $\%$ of the catalyst (excluding starch).

X-ray photoelectron spectroscopy (XPS) based on the Au (93$81 \mathrm{eV})$ and Pt $(80-68 \mathrm{eV})$ 4f signals provided $67 \pm 3 \mathrm{wt} \%$ of $\mathrm{Au} @ \mathrm{Pt} / \mathrm{G}-\mathrm{CB}$, Fig. S13, $\dagger$ with Pt comprising $21.8 \pm 0.7 \mathrm{wt} \%$ and $\mathrm{Au} 45 \pm 3 \mathrm{wt} \%$. More Pt was found with XPS, a surface specific technique, than EDX due to Pt-rich NP surface. The thermal treatment did not affect the $\mathrm{Au}$ and $\mathrm{Pt} 4 \mathrm{f}$ spectra, but the shoulder in the $\mathrm{C} 1 \mathrm{~s}$ spectrum at $286.5 \mathrm{eV}$ corresponding to $\mathrm{C}-\mathrm{O}$ and the $\mathrm{O} 1 \mathrm{~s}$ signal disappeared after treatment. X-ray powder diffraction (XRD) and TEM were used to verify that the thermal treatment did not compromise the bimetallic NP nanostructure as no change in the diffraction pattern was found, Fig. S14. $\dagger$ Evaluation of the crystallite size using the Scherrer equation on the metal peaks at 38 and $65^{\circ}$ confirmed that no line narrowing and crystallite growth had occurred. TEM further supported that the overall catalyst structure was stable during the starch removal process, Fig. 5, though slight agglomeration of the NPs was observed.

Further electrochemical analysis of the Au@Pt/G-CB surface was undertaken, Fig. 6a. The CVs of bimetallic catalysts match that of immobilized, unsupported Au@Pt NPs but provide six times higher currents attributed to the optimized dispersion in three dimensions on the G-CB. The commercial catalyst exhibits characteristic Pt response throughout the applied potential
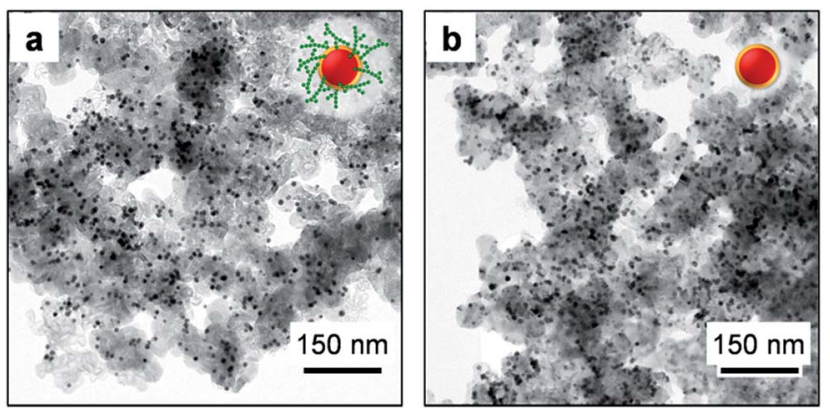

Fig. 5 TEM micrographs of (a) as-synthesized Au(Pt/G-CB catalyst and (b) Au@Pt/G-CB catalyst after thermal treatment to remove the starch coating.

range. The well-defined features of the supported catalysts allow for deeper evaluation of their electrochemical properties. The electrochemically active surface areas (ECSAs) of the Au@Pt/ G-CB catalysts and a commercial Pt/G-CB catalyst were evaluated by integrating the charge in the hydrogen adsorption/ desorption region (H-UPD) for Pt and the metal oxide reduction peaks for Pt and Au, Table 1 and Fig. S15. $\dagger$ The specific ECSA of $\mathrm{Pt}$ in the commercial catalyst was 21.2 and $21.8 \mathrm{~m}^{2} \mathrm{~g}^{-1}$ from $\mathrm{H}$-UPD and $\mathrm{PtO}_{x}$, respectively, which is consistent with previous reports. ${ }^{32}$ The specific ECSA of Pt in Au@Pt/G-CP was as high as 45.3-52.5 $\mathrm{m}^{2} \mathrm{~g}^{-1}, 2.1-2.4$ times that of the commercial catalyst depending on whether hydrogen adsorption or $\mathrm{PtO}_{x}$ reduction is considered, Table 1 . The Pt ECSA decreased drastically with thermal treatment (39\% from H-UPD and $33 \%$ from $\mathrm{PtO}_{x}$ ) which was unexpected from the XRD and TEM results. Indeed, the Au ECSA dropped by only $10 \%$ suggesting that agglomeration is not the only effect of heating. It is likely that the Pt shell rearranges forming thicker areas during heating. Considering the ECSAs from metal oxide reduction, the Pt coverage can be estimated by $\mathrm{ECSA}_{\mathrm{Pt}} / \mathrm{ECSA}_{\mathrm{Pt}+\mathrm{Au}}$. The Pt coverage decreases from 73 to $66 \%$ during thermal treatment due to contraction of the $\mathrm{Pt}$ shell. Braidy et al. showed with detailed EDX mapping how AuPt core-shell nanoparticles separate into pure phases joined by a flat interface at $600{ }^{\circ} \mathrm{C}$ in inert atmosphere. ${ }^{33}$ They reported that the core-shell structure is stable at $300{ }^{\circ} \mathrm{C}$. The smaller NP, thinner Pt shell, air atmosphere and catalysed starch oxidation in the current system could thus facilitate shell rearrangement at lower temperatures, e.g. $300{ }^{\circ} \mathrm{C}$. The increase in the $\mathrm{Au}: \mathrm{Pt}$ ratio after thermal treatment observed in XPS further supports that more $\mathrm{Au}$ is found at the particle surface. The greater reduction in Pt ECSA relative to Au ECSA could also be caused by partial dissolution of the Pt shell in the bulk of the NP. Both assynthesized and thermally treated Au@Pt/G-CB catalysts were further tested in catalytic systems.

\section{Electrocatalysis}

The Au@PtNP performance in FC anode electrocatalysis was evaluated by studying electrooxidation of small organic molecules used in direct alcohol/carboxylic acid FCs. The three tested systems vary in complexity from simple 2-electron oxidation of FA to the oxidation of EtOH in a 12-electron 

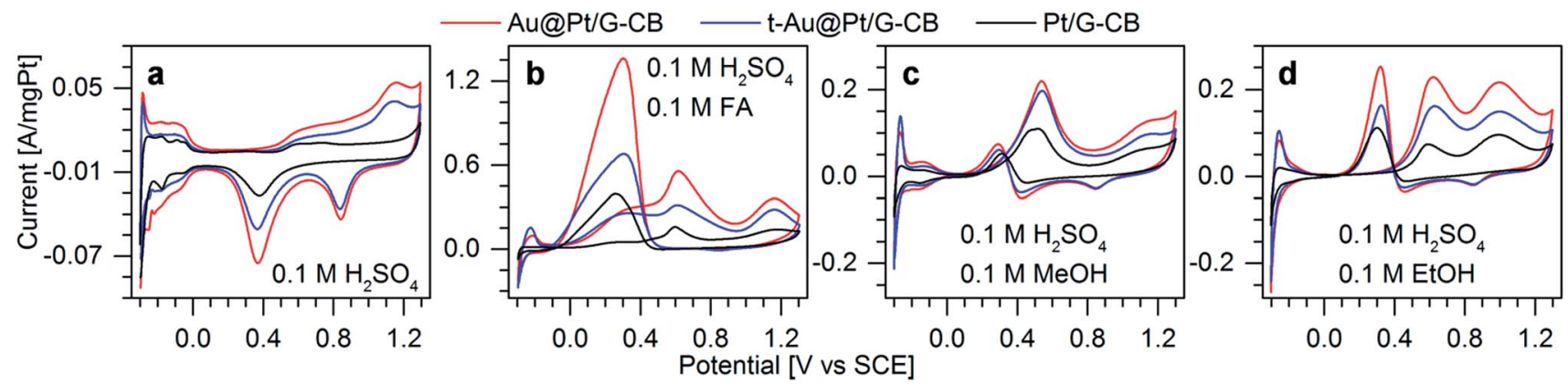

Fig. 6 Cyclic voltammograms of Au@Pt/G-CB (red), t-AuQPt/G-CB where starch has been removed (blue) and commercial Pt/G-CB (black) in (a) pure electrolyte $\left(0.1 \mathrm{M} \mathrm{H}_{2} \mathrm{SO}_{4}\right.$ ), or electrolyte with (b) $0.1 \mathrm{M}$ formic acid, (c) $0.1 \mathrm{M}$ methanol or (d) $0.1 \mathrm{M}$ ethanol. In all cases $\mathrm{RE}=\mathrm{RHE}$, $\mathrm{CE}=\mathrm{Pt}$ and scan rate $=50 \mathrm{mV} \mathrm{s}^{-1}$.

process. Fig. 6b-d shows CVs recorded at $50 \mathrm{mV} \mathrm{s}^{-1}$ in pure 0.1 $\mathrm{M} \mathrm{H}_{2} \mathrm{SO}_{4}$ electrolyte with $0.1 \mathrm{M} \mathrm{FA}$, MeOH or EtOH, respectively, Table 1. As-synthesized Au@Pt/G-CB, thermally treated Au@Pt/ G-CB without starch (t-Au@Pt/G-CB) and commercial PtNPs (Sigma-Aldrich) on G-CB (Pt/G-CB) were tested and are presented in Fig. 6 as red, blue and black lines, respectively. All data were recorded at identical Pt loadings and are shown as Pt mass specific activities.

Formic acid (FA) electrooxidation. Electrocatalytic oxidation of FA to $\mathrm{CO}_{2}$ on a metal surface may proceed via a triple path mechanism though the exact mechanism is under debate. ${ }^{2}$ Apart from direct decomposition of FA to $\mathrm{CO}_{2}$, oxidation can occur through dehydrogenation and adsorption of formate, or dehydration and subsequent oxidation of strongly adsorbed CO. ${ }^{34} \mathrm{CVs}$ of electrochemical oxidation of FA are shown in Fig. $6 \mathrm{~b}$ and display two anodic peaks at 0.30 and $0.61 \mathrm{~V} v s$. SCE corresponding to oxidation of FA via adsorbed formate and CO, respectively. ${ }^{32}$ The peak ratio, the pathway factor, indicates the catalyst selectivity towards direct oxidation through nonpoisoning dehydrogenation. The bimetallic catalysts exhibit higher pathway factors than the commercial PtNP catalyst and improved selectivity towards the formate pathway relative to pure Pt. The largest factor was observed for t-Au@Pt/G-CB more than 2.5 times that of Pt/G-CB. The mass activities estimated from the peak currents around $0.6 \mathrm{~V}$ were 556, 315 and $158 \mathrm{~mA}$ $\left(\mathrm{mg}_{\mathrm{Pt}}\right)^{-1}$ for Au@Pt/G-CB, t-Au@Pt/G-CB and Pt/G-CB, respectively, Table 1. The mass activity of the Au@Pt/G-CB is improved 3.5 times compared to the commercial catalyst and is higher than or comparable to several recently reported Pt-based catalysts such as $\mathrm{Pt}$ nanotetrahedra, ${ }^{35} \mathrm{PtCu}$ trigonal bipyramid nanoframes ${ }^{36}$ and PtPb nanoflowers, ${ }^{32}$ which were even obtained at higher FA concentrations (0.25-1 M) than those employed here. With a higher specific surface area of the bimetallic structures than of pure PtNPs, a higher mass activity is expected. Normalizing the CVs to Pt ECSA discloses a synergetic contribution from the Au core, Table 1, and a surface 55\% more active on the Au@Pt/G-CB compared to the Pt/G-CB. The surface of the thermally treated bimetallic catalyst was slightly less active $\left(1.02 \mathrm{~mA} \mathrm{~cm}^{-2}\right)$ than that of the as-synthesized one $\left(1.14 \mathrm{~mA} \mathrm{~cm}^{-2}\right)$ but still higher than for pure $\mathrm{Pt}\left(0.74 \mathrm{~mA} \mathrm{~cm}^{-2}\right)$. The $\mathrm{Au}$ core thus improves the $\mathrm{Pt}$ utilization not only by increasing active surface area but also by modifying the activity itself of that surface.

Methanol (MeOH) electrooxidation. The electrochemical oxidation of $\mathrm{MeOH}$ on $\mathrm{Pt}$ involves several intermediate steps, i.e. dehydrogenation, CO-like species chemisorption, $\mathrm{OH}^{-}$(or $\mathrm{H}_{2} \mathrm{O}$ ) adsorption, chemical interaction between adsorbed $\mathrm{CO}$ and -OH compounds, and $\mathrm{CO}_{2}$ evolution. The primary by-products in methanol oxidation are formaldehyde and FA. ${ }^{37}$ Electrooxidation of $\mathrm{MeOH}$ is a 6-electron process which gives rise to the formation of strongly adsorbed $\mathrm{CO}$ species in linear or bridgebonded form, i.e. catalyst poisoning. ${ }^{37}$ The electrocatalytic activity of Pt can be promoted by the presence of a second metal (e.g. $\mathrm{Ru}, \mathrm{Sn}, \mathrm{Au})^{38}$ acting either as an adatom or a bimetal. CVs in Fig. $6 \mathrm{c}$ show the electrooxidation of $\mathrm{MeOH}$ on the three tested catalysts. One main anodic peak is observed at $0.54 \mathrm{~V}$ in the forward scan and represents oxidation of $\mathrm{MeOH}$ through dehydrogenation and adsorption of residues. ${ }^{39}$ Again, the assynthesized Au@Pt/G-CB demonstrated the highest massspecific peak-current density of $219 \mathrm{~mA}\left(\mathrm{mg}_{\mathrm{Pt}}\right)^{-1}$, or twice that of

Table 1 Electrochemical characterization and fuel oxidation efficiency of Pt-based catalysts

\begin{tabular}{|c|c|c|c|c|c|c|c|c|c|c|}
\hline \multirow[b]{2}{*}{ Catalyst } & \multicolumn{3}{|c|}{$\operatorname{ECSA}\left[\mathrm{m}^{2} \mathrm{~g}^{-1}\right]$} & \multirow[b]{2}{*}{ Coverage $\Gamma_{\mathrm{Pt}}{ }^{a}$} & \multicolumn{3}{|c|}{$\underline{\text { Mass activity }\left[\mathrm{mA} \mathrm{mg}_{\mathrm{Pt}}{ }^{-1}\right]}$} & \multicolumn{3}{|c|}{ Surface area activity ${ }^{b}\left[\mathrm{~mA} \mathrm{~cm}^{-2}\right]$} \\
\hline & H-UPD & $\mathrm{PtO}_{x}$ & $\mathrm{AuO}_{x}$ & & FAOR, $0.61 \mathrm{~V}$ & MOR, $0.54 \mathrm{~V}$ & EOR, $0.62 \mathrm{~V}$ & FAOR, $0.61 \mathrm{~V}$ & MOR, $0.54 \mathrm{~V}$ & EOR, $0.62 \mathrm{~V}$ \\
\hline t-Au@Pt/G-CB & 27.5 & 35.1 & 5.85 & $66 \%$ & 315 & 197 & 162 & 1.02 & 0.64 & 0.53 \\
\hline $\mathrm{Pt} / \mathrm{G}-\mathrm{CB}$ & 21.2 & 21.8 & - & $(100 \%)$ & 158 & 110 & 74 & 0.74 & 0.51 & 0.34 \\
\hline
\end{tabular}

${ }^{a}$ Pt surface area relative to total metal surface area based on metal oxide reduction. ${ }^{b}$ Data normalized to the average ECSA from H-UPD and PtO . $^{\circ}$ 
the commercial Pt catalyst $\left(110 \mathrm{~mA}\left(\mathrm{mg}_{\mathrm{Pt}}\right)^{-1}\right)$. This value is higher than obtained previously at 5-times higher fuel concentration with $\mathrm{Au}-\mathrm{Pt}$ core-shell systems. ${ }^{\mathbf{4 0}}$ Recently, currents around $500 \mathrm{~mA}\left(\mathrm{mg}_{\mathrm{Pt}}\right)^{-1}$ in $1 \mathrm{M} \mathrm{MeOH}$ with $\mathrm{Au}-\mathrm{Pt}$ core-shell NPs on carbon support, ${ }^{41}$ PtRu NPs on N-doped graphene ${ }^{42}$ and $\mathrm{Pt}$ NPs on $\mathrm{TiO}_{2} / \mathrm{N}$-doped carbon nanocomposite were obtained. $^{43}$ Peak-currents increase with increasing $\mathrm{MeOH}$ concentration. ${ }^{44}$ In contrast to FA oxidation, the thermally treated catalyst showed only slight reduction in activity (197 mA $\left.\left(\mathrm{mg}_{\mathrm{Pt}}\right)^{-1}\right)$ indicating that reduction of ECSA induced by heating was counteracted by increased activity towards the MOR. Indeed, the surface activity was highest for t-Au@Pt/G-CB at $0.64 \mathrm{~mA} \mathrm{~cm}^{-2}$, or $25 \%$ higher than for pure Pt. The surface of the as-synthesized Au@Pt/G-CB was less active for MOR than the commercial Pt catalyst. These observations further support that the chemistry of the Pt shell is modified by interaction with the Au core affecting the $\mathrm{MeOH}$ electrooxidation.

Ethanol (EtOH) electrooxidation. EtOH electrooxidation leads to several products, acetic acid, acetaldehyde, acetyl radical, $\mathrm{CO}$ and $\mathrm{CO}_{2}$. Its complete oxidation delivers 12 electrons per EtOH molecule giving very high energy density. ${ }^{\mathbf{1 4}}$ However, breaking $\mathrm{C}-\mathrm{C}$ bonds is difficult and $\mathrm{CO}_{2}$ is only produced to a small extent in electrochemical oxidation. ${ }^{\mathbf{1 4}}$ Several spectroscopic and electrochemical techniques have been applied to elucidate the mechanism of EtOH electrooxidation on Pt-based materials. ${ }^{45,46}$ The contributions of the different pathways depend strongly on EtOH concentration. ${ }^{47}$ Above $0.1 \mathrm{M}$, less water is present at the surface and formation of $\mathrm{CO}_{2}$ and acetic acid is significantly inhibited, while production of acetaldehyde starts to increase. ${ }^{47}$

The activity of the three catalysts towards the EOR is illustrated by the CVs in Fig. $6 \mathrm{~d}$ showing a main oxidation peak at
$0.62 \mathrm{~V}$ and a second peak at $1.0 \mathrm{~V}$ which is due to further oxidation of intermediate products. ${ }^{47}$ The catalyst activities follow the same trend as for the FAOR with mass-specific peak currents of Au@Pt/G-CB (229 mA $\left.\left(\mathrm{mg}_{\mathrm{Pt}}\right)^{-1}\right)$ and t-Au@Pt/G-CB (162 $\mathrm{mA}\left(\mathrm{mg}_{\mathrm{Pt}}\right)^{-1}$ ) being 3.1 and 2.2 times higher than for commercial Pt/G-CB $\left(74 \mathrm{~mA}\left(\mathrm{mg}_{\mathrm{Pt}}\right)^{-1}\right)$. As with the MOR, the Pt ECSA-specific activity was highest for the thermally treated catalyst, t-Au@Pt/G-CB followed by the as-synthesized Au@Pt/ G-CB being 55\% and 38\% higher than Pt/G-CB, respectively (Table 1). Notably the ratio between the 0.62 and $1.0 \mathrm{~V}$ peaks for $\mathrm{Au} @ P t$ catalysts was opposite that of Pt/G-CB indicating modification of surface specificity for EtOH oxidation in the presence of the Au core. This is supported by a recent theoretical study in which EtOH and acetyl radical were found to exhibit closer binding interactions on $\mathrm{Pt} / \mathrm{Au}(111)$ than on pure $\mathrm{Pt}(111) .{ }^{48}$

\section{Catalytic hydrogenation of benzenes}

The activity of Au@Pt NPs towards hydrogenation (reduction) reactions was probed using substituted benzenes and tested in the hydrocyclization of LA to GVL, Scheme S2. $\dagger$ ARH of BM to cyclohexane carboxamide (CCM) was chosen as a standard reaction to optimize the reaction parameters, such as solvent effects and thermal pre-treatment, before testing BA, and LA/MLA hydrogenation. The Au@Pt/G-CB was screened in its assynthesized and thermally treated forms using both protic (water) and aprotic (toluene) solvents to study how the solvent environment affects the catalyst activity. Fig. 7 (and Table S4†) shows the catalysts activity for chemoselective hydrogenation of BM to CCM in both water and toluene using $\mathrm{H}_{2}$ as reducing agent. The Au@Pt catalyst was active and highly selective for BM hydrogenation under all reaction conditions. The thermally treated catalyst (Fig. 7III) showed no superior activity over the

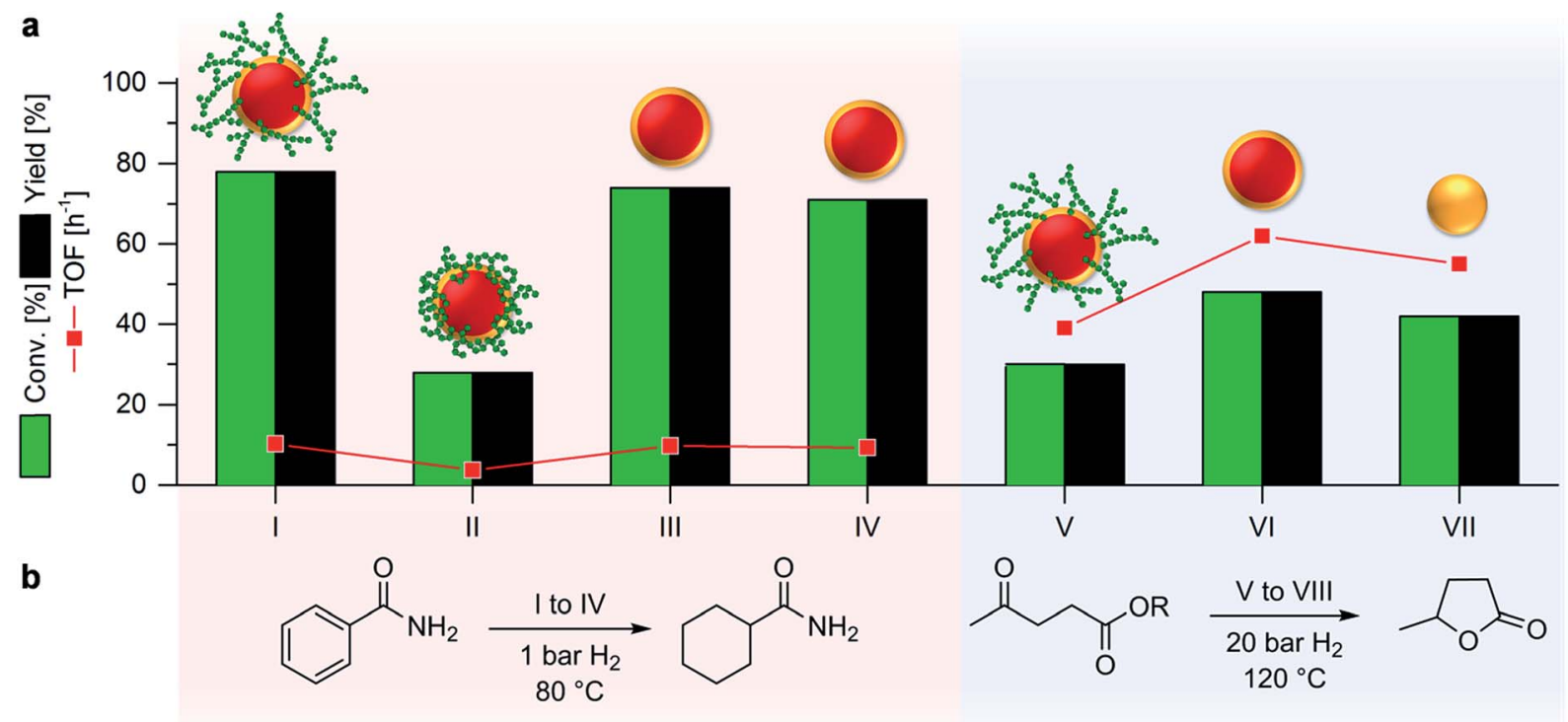

Fig. 7 (a) Conversion (green), yield (black) and TOF (red) for catalytic hydrogenation of $0.5 \mathrm{mmol}$ BM (I to IV), $0.5 \mathrm{~mol}$ MLA (V to VII). Schemes of catalyst NP structures are shown on top of the columns. (b) Reaction schemes and general conditions for BM and MLA hydrogenation. Catalyst: (I, II, and V) as-synthesized Au@Pt/G-CB, (III, IV, and VI) thermally treated Au@Pt/G-CB, (VII) commercial Pt/G-CB. Catalyst loading: (I-IV) 0.32 mol\% Pt, (V-VII) 1.5 mol\% Pt. Solvent: (I, III, V-VII) water, (II and IV) toluene. Reaction time: (I-IV) 24 h, (V-VII) 30 min. 
as-synthesized catalyst in water (I). Instead, a slight decrease (from 78 to $71 \%$ CCM yield) was noticed, most likely due to the nanostructural changes induced by the thermal treatment, as discussed previously. The activity of as-synthesized Au@Pt/ G-CB dropped to $28 \%$ when going from water to toluene (II), but could be recovered to nearly that of water by removing starch (IV). This is due to the low solubility of starch in toluene, Fig. S16. $\uparrow$ The non-hydrated starch molecules on the Pt surface in non-polar environment do not extend into the solution but rather collapse resulting in limited access to the active sites. The hydrogenation rate can be related to H-bond donation of the environment around the active site. A lower activation energy barrier for protic solvents, due to strong interaction between protic solvents and substrate by hydrogen bonding, leads to higher hydrogenation rates. ${ }^{49}$ This is of minor importance in the present system where the activity of thermally treated catalyst is similar in both solvents (III and IV). Finally, it is worth noting that no hydrogenation product of toluene was observed.

The Au@Pt/G-CB catalyst showed also excellent catalytic activity for chemoselective hydrogenation of BA under mild conditions. Quantitative yields (100\%) of CCA were achieved with $>99 \%$ selectivity in $24 \mathrm{~h}$ at $1 \mathrm{~atm} \mathrm{H}_{2}$ pressure and $80{ }^{\circ} \mathrm{C}$. $\mathrm{N}$-doped carbon supported $\mathrm{Pd}$ nanoparticles was recently shown to bring a nine-fold increase in activity compared to commercial Pd on activated carbon for the hydrogenation of BA under similar conditions. ${ }^{49}$ The Au@Pt/G-CB introduced in this work provided as much as 15-fold increased turn-over frequency (TOF) at $0.3 \mathrm{~mol} \% \mathrm{Pt}$ with $\mathrm{Au}(1.0 \mathrm{~mol} \%)$. No traces of BA hydrogenation to benzyl alcohol were observed under the given reaction conditions and CCA was the only product.

\section{Hydrocyclization of LA to GVL}

Carbon supported Pt catalysts have previously shown poor activity and over-hydrogenation of GVL resulting in low selectivity. ${ }^{27,50}$ Improved activity was reported by Kon et al. with $\mathrm{Pt} / \mathrm{C}$ resulting in $14 \%$ yield of GVL in $1 \mathrm{~h}$ at relatively low pressure ( 8 bars) and high temperature $\left(200{ }^{\circ} \mathrm{C}\right) .{ }^{51} \mathrm{Au} @ \mathrm{Pt} / \mathrm{G}-\mathrm{CB}$ was screened for LA hydrogenation followed by cyclization to GVL. Quantitative yields of GVL were attained with excellent selectivity (> 99\%) (Table S5, VIII $\dagger$ ). The commercial Pt/G-CB catalyst was applied as a reference providing similar results. To determine the TOF, shorter reaction times were chosen. Reaction with commercial Pt/G-CB for $5 \mathrm{~h}$ gave quantitative yields of GVL at a TOF of $13 \mathrm{~h}^{-1}$ (Table S5, IX $\dagger$ ). The activities of Au@Pt NP and commercial Pt catalysts (Fig. 7V-VII) were compared at 30 min reaction times. Commercial Pt/G-CB catalyst yielded $40 \%$ more GVL than as-synthesized Au@Pt/G-CB but the highest yields were obtained with thermally treated Au@Pt/G-CB. The removal of the starch capping thus resulted in a significantly higher catalytic activity than that of commercial Pt/G-CB (48\% yield $v s .42 \%$ ) at a TOF of $62 \mathrm{~h}^{-1}$. This observation highlights the need for starch removal for LA hydrocyclization. The larger molecular size of LA relative to other tested substrates (FA, $\mathrm{MeOH}, \mathrm{EtOH}, \mathrm{BA}, \mathrm{BM}$ ) may hinder diffusion through the starch layer and limit the activity. The surface structure changes induced by the heating might also increase the activity towards LA hydrocyclization. All the catalysts showed excellent selectivity to GVL with no detectable over-hydrogenation (formation of valeric acid, 2-Me-THF).

\section{Conclusions}

The green synthesis protocol SAMENS was developed for synthesis of highly active starch protected $8 \pm 2 \mathrm{~nm}$ core-shell NPs (Au@PtNPs) consisting of a spherical Au core and a $3 \AA$ atomically thin Pt shell. The G-CB supported Au@Pt catalyst (3 wt\% Pt, $9 \mathrm{wt} \% \mathrm{Au}$ ) was thoroughly characterized. Starch removal by heating changed the chemistry of the Pt shell as seen in the electrochemical characterization, and strongly affected the catalytic performance in electrochemical oxidation and hydrogenation, which possibly relate to structural changes of the Pt shell. Catalytic activities for several electrooxidation and hydrogenation reactions were investigated and compared to commercial Pt/G-CB. The high specific Pt surface area of the thin Pt shell led to superior activity of Au@Pt/G-CB over Pt/G-CB for electrochemical oxidation of important FC fuels (FA, $\mathrm{MeOH}$ and EtOH). Au@Pt/G-CB mass activities (in mA $\left(\mathrm{mg}_{\mathrm{Pt}}\right)^{-1}$ ) of 556 for FAOR, 219 for MOR and 229 for EOR were 3.5, 2.0 and 3.1 times higher than for the commercial Pt/G-CB catalyst. The core-shell structure gave synergy between the Pt shell and the Au core, as well as limiting inactive Pt. The activity of the Pt surface increased with up to $55 \%$.

The presence of the starch coating had insignificant effects on the catalytic activity for ARH of BM in water. Removal of starch prompted a 2.5-fold increase in activity in toluene in which the hydrophilic starch chains collapse, passivating the surface. The Au@Pt/G-CB catalyst was active for GVL production from LA/MLA via hydrogenation/cyclization. For this system, the highest activity (TOF $=62 \mathrm{~h}^{-1}$ ) was exhibited by the thermally treated catalyst.

The facile, green and robust synthesis presented here produces highly active and well-defined bimetallic nanostructures and offers prospects of further tuning catalytic activity/selectivity by the Au core size, Pt shell thickness and thermal treatment.

\section{Acknowledgements}

Assistance with STEM-EDX experiments from Wilhelmus Huyzer (DTU CEN), TGA measurements from Larisa Seerup (DTU Energy Conversion and Storage) and the experimental hydrogenation setup from Eduardo J. García-Suárez (DTU Chemistry) are appreciated. Financial support from Danish Council for Independent Research Technology and Production Sciences (DFF-1335-00330) and the Lundbeck Foundation (R141-2013-13273) are acknowledged.

\section{Notes and references}

1 N. Tian, J. Xiao, Z. Zhou, H. Liu, Y. Deng, L. Huang, B. Xu and S. Sun, Faraday Discuss., 2013, 162, 77-89. 
2 K. Jiang, H. Zhang, S. Zou and W. Cai, Phys. Chem. Chem. Phys., 2014, 16, 20360-20376.

3 R. G. Chaudhuri and S. Paria, Chem. Rev., 2012, 112, 2373-2433. 4 M. Daniel and D. Astruc, Chem. Rev., 2004, 104, 293-346.

5 A. Villa, D. Wang, D. S. Su and L. Prati, Catal. Sci. Technol., 2015, 5, 55-68.

6 B. N. Wanjala, J. Luo, R. Loukrakpam, B. Fang, D. Mott, P. N. Njoki, M. Engelhard, H. R. Naslund, J. K. Wu, L. Wang, O. Malis and C. Zhong, Chem. Mater., 2010, 22, 4282-4294.

7 D. F. Yancey, E. V. Carino and R. M. Crooks, J. Am. Chem. Soc., 2010, 132, 10988-10989.

8 J. Luo, L. Wang, D. Mott, P. N. Njoki, Y. Lin, T. He, Z. Xu, B. N. Wanjana, I.-S. Lim and C. Zhong, Adv. Mater., 2008, 20, 4342-4347.

9 C. J. Serpell, J. Cookson, D. Ozkaya and P. D. Beer, Nat. Chem., 2011, 3, 478-483.

10 C. J. Murphy, J. Mater. Chem., 2008, 18, 2173-2176.

11 C. Engelbrekt, K. H. Sorensen, J. Zhang, A. C. Welinder, P. S. Jensen and J. Ulstrup, J. Mater. Chem., 2009, 19, 78397847.

12 C. Engelbrekt, P. S. Jensen, K. H. Sorensen, J. Ulstrup and J. Zhang, J. Phys. Chem. C, 2013, 117, 11818-11828.

13 C. Engelbrekt, K. H. Sorensen, T. Lubcke, J. Zhang, Q. Li, C. Pan, N. J. Bjerrum and J. Ulstrup, ChemPhysChem, 2010, 11, 2844-2853.

14 G. L. Soloveichik, Beilstein J. Nanotechnol., 2014, 5, 13991418.

15 S. Patton, T. Cropp and K. Reynolds, Biochemistry, 2000, 39, 7595-7604.

16 B. Moore, H. Cho, R. Casati, E. Kennedy, K. Reynolds, U. Mocek, J. Beale and H. Floss, J. Am. Chem. Soc., 1993, $115,5254$.

17 H. Weissermel and H. J. Arpe, Industrial Organic Chemistry, Wiley-VCH, Weinheim, 1997.

18 J. Thomas, B. Johnson, R. Raja, G. Sankar and P. Midgley, Acc. Chem. Res., 2003, 36, 20-30.

19 H. Wang and F. Zhao, Int. J. Mol. Sci., 2007, 8, 628-634.

20 B. Zong, X. Zhang and M. Qiao, AIChE J., 2009, 55, 192-197.

21 R. Raja, T. Khimyak, J. Thomas, S. Hermans and B. Johnson, Angew., Chem. Int. Ed., 2001, 40, 4638-4642.

22 SNIA VISCOSA, 1968, IE 1122162.

23 SNIA VISCOSA, 1966, IE 1019795.

24 SNIA VISCOSA, 1964, IE 967918.

25 C. Tsung, J. N. Kuhn, W. Huang, C. Aliaga, L. Hung, G. A. Somorjai and P. Yang, J. Am. Chem. Soc., 2009, 131, 5816-5822.

26 D. W. Rackemann and W. O. S. Doherty, Biofuels, Bioprod. Biorefin., 2011, 5, 198-214.
27 L. Manzer, Appl. Catal., A, 2004, 272, 249-256.

28 P. P. Upare, J. Lee, D. W. Hwang, S. B. Halligudi, Y. K. Hwang and J. Chang, J. Ind. Eng. Chem., 2011, 17, 287-292.

29 N. G. Bastus, J. Comenge and V. Puntes, Langmuir, 2011, 27, 11098-11105.

30 A. Henglein, J. Phys. Chem. B, 2000, 104, 2201-2203.

31 L. N. Cleemann, F. Buazar, Q. Li, J. O. Jensen, C. Pan, T. Steenberg, S. Dai and N. J. Bjerrum, Fuel Cells, 2013, 13, 822-831.

32 M. Gong, F. Li, Z. Yao, S. Zhang, J. Dong, Y. Chen and Y. Tang, Nanoscale, 2015, 7, 4894-4899.

33 N. Braidy, G. R. Purdy and G. A. Botton, Acta Mater., 2008, 56, 5972-5983.

34 M. Neurock, M. Janik and A. Wieckowski, Faraday Discuss., 2008, 140, 363-378.

35 M. Rana, M. Chhetri, B. Loukya, P. K. Patil, R. Datta and U. K. Gautam, ACS Appl. Mater. Interfaces, 2015, 7, 49985005.

36 S. Chen, H. Su, Y. Wang, W. Wu and J. Zeng, Angew., Chem. Int. Ed., 2015, 54, 108-113.

37 A. S. Arico, S. Srinivasan and V. Antonucci, Fuel Cells, 2001, 1, 133-161.

38 A. Hamnett, Catal. Today, 1997, 38, 445-457.

39 S. Wasmus and A. Kuver, J. Electroanal. Chem., 1999, 461, 1431.

40 S. Wang, N. Kristian, S. Jiang and X. Wang, Nanotechnology, 2009, 20, 025605.

41 S. Yan, S. Zhang, W. Zhang, J. Li, L. Gao, Y. Yang and Y. Gao, J. Phys. Chem. C, 2014, 118, 29845-29853.

$42 \mathrm{X} . \mathrm{Xu}, \mathrm{Y} . \mathrm{Zhou}, \mathrm{J}$. Lu, X. Tian, H. Zhu and J. Liu, Electrochim. Acta, 2014, 120, 439-451.

43 X. Zhao, J. Zhu, L. Liang, J. Liao, C. Liu and W. Xing, J. Mater. Chem., 2012, 22, 19718-19725.

44 X. Wang, W. Wang, Z. Qi, C. Zhao, H. Ji and Z. Zhang, J. Power Sources, 2010, 195, 6740-6747.

45 J. de Souza, S. Queiroz, K. Bergamaski, E. Gonzalez and F. Nart, J. Phys. Chem. B, 2002, 106, 9825-9830.

46 S. Sen Gupta and J. Datta, J. Chem. Sci., 2005, 117, 337344.

47 G. Camara and T. Iwasita, J. Electroanal. Chem., 2005, 578, 315-321.

48 A. O. Pereira and C. R. Miranda, Appl. Surf. Sci., 2014, 288, 564-571.

49 X. Xu, M. Tang, M. Li, H. Li and Y. Wang, ACS Catal., 2014, 4, 3132-3135.

50 A. M. Hengne, N. S. Biradar and C. V. Rode, Catal. Lett., 2012, 142, 779-787.

51 K. Kon, W. Onodera and K. Shimizu, Catal. Sci. Technol., 2014, 4, 3227-3234. 